\title{
TREM2 is a prognostic biomarker and correlates in immune infiltrates in kidney renal papillary cell carcinoma and liver hepatocellular carcinoma
}

bin wen Xiang

Wuhan University Zhongnan Hospital

Sha Fang

Wuhan University Zhongnan Hospital

peng yan Ding

Wuhan University Zhongnan Hospital

min nuo Liu

Wuhan University Zhongnan Hospita

chao hua Wu

Wuhan University Zhongnan Hospital

Haijun Yu ( $\nabla$ haijunyu@whu.edu.cn )

Wuhan University Zhongnan Hospital https://orcid.org/0000-0003-1943-2770

Research

Keywords: TREM2, kidney renal papillary cell carcinoma (KIRP), prognosis, immune infiltrates

Posted Date: February 16th, 2021

DOI: https://doi.org/10.21203/rs.3.rs-170595/v1

License: (9) (i) This work is licensed under a Creative Commons Attribution 4.0 International License.

Read Full License 


\title{
TREM2 is a prognostic biomarker and correlates in immune
} infiltrates in kidney renal papillary cell carcinoma and liver

\section{hepatocellular carcinoma}

\begin{abstract}
Background: TREM2 was identified as a marker for tumor-associated macrophages (TAMs) and monocytes. However, there is limited information concerning the TREM2 mRNA levels correlated with the outcome and tumor-infiltrating lymphocytes in different cancers.

Methods: To explore the expression pattern and prognostic value of TREM2 in pan-cancer, We use multiple databases, including ONCOMINE, PrognoScan, Kaplan-Meier Plotter, GEPIA, and TIMER. we investigated the correlations between TREM1 expression and immune infiltration in cancers, especially kidney renal papillary cell carcinoma (KIRP), and liver hepatocellular carcinoma (LIHC)
\end{abstract}

Results: According to the results from Oncomine and TIMER datasets, in a general way, TREM2 mRNA expression is higher in the majority of the tumor and is lower in lung cancer, compared with normal adjacent tissues. A high expression level of TREM2 was beneficial to the survival of LIHC patients. Both in KIRP and LIHC, TREM2 expression levels showed significant positive correlations with infiltrating levels of macrophages and dendritic cells. It also correlated with diverse immune marker sets. We can also find that TREM2 expression was modestly associated with CD8+T cells in KIRP. However, it is related to the Treg in LIHC.

Conclusions: TREM2 may be a prognostic marker in the multitudinous tumor. Furthermore, TREM2 may interact with immune infiltration to influence patient survival in cancers like LIHC and KIRP. 
Keywords: TREM2, kidney renal papillary cell carcinoma (KIRP), prognosis, immune infiltrates

\section{Introduction}

Papillary renal cell carcinoma (PRCC) accounts for 10-15\% of renal clear cell carcinoma, Which

is the most common malignancy among both men and women worldwide[1]. The number of people suffering from Papillary renal cell carcinoma each year increases to 6-9000. In renal clear cell carcinoma, immune-related mechanisms play an essential role, and immuneoncology strategies can be seen as a promising path for renal clear cell carcinoma[2]. However, most of the FDA proved therapeutic drugs are for CCRCC[3], Mainly targeted therapy (antiangiogenesis), plus the old immunotherapy interleukin-2 has been tested on papilloma, but with little success[4, 5]. Moreover, more and more studies have shown that the prognosis and effectiveness of chemotherapy and immunotherapy are impaired by tumor-infiltrating lymphocytes, such as tumor-associated macrophages and tumor-infiltrating neutrophils. There is an urgent need to explain the immune phenotype of tumor-immune interactions in Papillary renal cell carcinoma and develop new immune-related therapeutic targets.

TREM2 is one of the Ig-superfamily members. When TREM2 binds to an adaptor protein DAP12, activation signaling is transmitted. The function and pathological significance of trem2 have been extensively studied in non-cancerous diseases, such as Alzheimer's disease[6, 7]. in promoting the immune-suppressive microenvironment of cancer tumors. An analysis of 
gastric cancer samples observed that TREM2 mRNA and protein expression were substantially higher relative to normal gastric tissues. The high expression of Trem2 predicted poor prognosis[9]. TREM2 has been proposed as a target for Glioma[10] and liver cancer[11] because its upregulation is associated with tumor progression. Recently, Katzenelenbogen et al. Give sufficient evidence to illustrate that trem2 is a marker for tumor-associated macrophages (TAMs) and monocytes[12]. It attracted our attention to the tumor inhabitation role and mechanism of Trem2. Whether tumor immune infiltrates and clinical effects are correlated with trem2 levels in Papillary renal cell carcinoma tissues has not been evaluated. Our study comprehensively evaluated the prognostic landscape of TREM2 in databases such as Oncomine, PrognoScan, and Kaplan-Meier plotter. Through the Tumor Immune Estimation Resource (TIMER) and GEPIA, we investigated the association of time with tumor-infiltrating immune cells in various tumor microenvironments. Our findings shed light on the crucial role of Trem2 in Papillary renal cell carcinoma and provide a potential relationship and an underlying mechanism between Papillary renal cell carcinoma and tumor-immune interactions.

\section{Materials \& Methods}

\section{Expression of trem2 in human tumors}

The expression level of the TREM2 gene in various types of cancers was analyzed in the Oncomine database(https://www.oncomine.org/resource/login.html)[13]. The thresholds were set as a P-value of 0.001 , fold change of 1.5 , and gene ranking of all. Survival analysis 
The correlation between The mRNA expression of TREM2 and survival in different cancer types of cancers was analyzed by the PrognoScan database (http://dna00.bio.kyutech.ac.jp/PrognoScan/index.html)[14], GEPIA(http://gepia.cancerpku.cn/)[15], and Kaplan-Meier Plotter(https://kmplot.com/analysis/)[16].PrognoScan looks through a wide range of freely accessible cancer microarray databases for determining relationships between gene expression and patient prognosis, such as overall survival (OS) and disease-free survival (DFS). The threshold was set as a Cox P-value $<0.05$. Besides, we downloaded TREM2 RNAseq data and clinical data of GDC TCGA Kidney Papillary Cell Carcinoma (KIRP) from UCSC-XENA(https://xenabrowser.net/datapages/)[17] and integrated the data via EXCEL. We did univariate survival analysis via GraphPad. The survival analysis from PrognoScan was summarized and visualized via forest map was drawn by GraphPad and adobe illustrator.

GEPIA, an interactive web server, is usually utilized to analyze the RNA sequencing expression data, including 9,736 tumors and 8,587 normal samples from the TCGA and the GTEx projects. We generate survival curves in each available cancer type (total number=34), including OS and DFS, with the Spearman method determining the correlation coefficient.

The Kaplan-Meier plotter can identify the effect of 54000 genes on survival in 21 tumors. We explored the impact of trem2 expression on OS and RFS and calculated Ratios (HRs) with 95\% confidence intervals $(\mathrm{Cl})$ and log-rank $\mathrm{P}$ values.

Trem2 expression and immune cell associations in TIMER and GEPIA

We determine the relationship between Trem2 expression and immune infiltration via TIMER (https://cistrome.shinyapps.io/timer/)[18] and GEPIA databases. TIMER is a powerful online 
tool that does a systematic analysis of immune infiltrates across diverse cancer types. a previously published statistical deconvolution method is applied to infer the abundance of tumor-infiltrating immune cells from gene expression profiles. 10,897 samples across 32 cancer types from The Cancer Genome Atlas (TCGA) are contained to estimate immune infiltrates quantity. We analyzed the correlation of trem2 expression with infiltrating cells the abundance of immune infiltrates, including B cells, CD4+ T cells, CD8+ T cells, neutrophils, macrophages, and dendritic cells and also determined the relationship of gene expression levels with tumor purity. To identify the potential subtypes of infiltrating immune cells. We further analyzed the correlation between TREM2 expression and several immune cell markers. These markers are determined by referencing studies previously, respectively, markers of CD8+ T cells, T cells (general), B cells, monocytes, TAMs, M1 macrophages, M2 macrophages, neutrophils, natural killer (NK) cells, dendritic cells (DCs), T-helper 1 (Th1) cells, T-helper 2 (Th2) cells, follicular helper T (Tfh) cells, T-helper 17 (Th17) cells, Tregs, and exhausted T cells, Log2 RSEM was used to be the standard of adjusting the gene expression level. Correlations between trem 2 and each immune gene marker were displayed via scatter plots. The plots include Spearman's correlation and the estimated statistical significance. Also, trem2 as a gene symbol is for the $\mathrm{x}$-axis, and related marker genes are plotted on the $\mathrm{y}$-axis as gene symbols.

\section{Statistical analysis}

The results from Oncomine datasets are seen with P-values, fold changes, and ranks. As for the survival curves, the log-rank test was the standard method to calculate HR and log-rank P-value in Kaplan-Meier Plotter and GEPIA., while in PrognoScan, HR and Cox P value was 
calculated by a Univariate Cox regression model. Spearman's correlation was used to

112 calculate the gene expression correlation, if not explicitly stated, P-values $<0.05$ is deemed 113 statistically significant.

114

\section{Results}

The mRNA expression levels of TREM2

Comparing with that in the respective normal groups, TREM2 is highly expressed in bladder cancer, brain and CNS cancer, breast cancer, cervical cancer, colorectal cancer, esophageal cancer, stomach cancer, head and neck cancer, kidney cancer, pancreatic cancer, and prostate cancer. Besides, TREM2 expression is lower in lung cancer compared to normal tissues(Figure 1A). We summarized the detailed results of TREM2expression in multiple cancers in Supplementary Table 1.

To further evaluate TREM2 expression in human cancers, we used the RNA-seq data of multiple malignancies in TCGA, examining TREM2expression(Figure 1B). TREM2 expression was significantly higher in BLCA(bladder urothelial carcinoma)、 BRCA(breast invasive carcinoma)、CESC(Cervical squamous cell carcinoma and endocervical adenocarcinoma)、 $\mathrm{CHOL}$ (Cholangiocarcinoma)、 $\mathrm{COAD}$ (colon adenocarcinoma)、GBM(Glioblastoma multiforme)、 HNSC(head and neck cancer)、 $\mathrm{KICH}$ (kidney chromophobe)、 KIRC(kidney renal clear cell carcinoma)、 KIRP(Kidney renal papillary cell carcinoma)、 LIHC(liver hepatocellular carcinoma)、 PRAD(prostate adenocarcinoma) 、 STAD(stomach adenocarcinoma) 、 THCA(thyroid carcinoma), UCEC(uterine corpus endometrial carcinoma). Besides that, the expression of TREM2 was lower in LUAD(lung adenocarcinoma)、 LUSC(lung squamous cell carcinoma) than 
in their respective adjacent normal tissues.

\section{The Relationship between TREM2 and prognosis in pan-cancer}

We evaluate the expression of TREM2 via three datasets. Firstly, in PrognoScan, the results showed that in 3 types of cancers, including lung, colorectal, and breast cancers, TREM2 expression significantly influences prognosis(Figure 2A-H). Among them, TREM2 played a detrimental role in Colorectal cancer (DFS: HR=1.23, $\operatorname{coxp}=0.026$; DFS: HR=2.07, Coxp=0.046). Meanwhile, an interesting result can be noticed in lung cancer and breast cancer. ONE cohorts (GSE31210) included 204 samples showed that low expression was notably associated with better prognosis(OS HR=2.21, Coxp $=0.0014$; RFS HR=1.88, $\operatorname{coxp}=<0.001$.), however, three cohorts(GSE1321,GSE11117,GSE13213)suggest the reverse may be the case(OS HR=0.37, Cox $p=<0.001$; OS HR=0.55, $\operatorname{coxp}=0.008$; OS HR=0.72, $\operatorname{Coxp}=0.019)$. the opposite results can also be seen in the breast cancer. One cohort (GSE1379) that included 60 samples showed that high expression was notably associated with poor RFS (RFS HR=2.15, $\operatorname{coxp}=0.044$ ), and another cohort (GSE19615) showed the opposite result. (DMFS HR=0.32, Coxp=0.029). Therefore, it is conceivable that low TREM2expression is an independent risk factor and leads to a better prognosis in CRC patients.

To further examine the prognostic potential of TREM2 in different cancers, we evaluate the TREM2 prognostic value based on Affymetrix microarrays via the Kaplan-Meier plotter database. These results suggested that low TREM2 expression was significantly associated with better prognosis in Ovarian(OS HR $=1.27(1.09-1.47)$ log-rank $P=0.0014 ;$;FS HR $=$ 

1.25 (1.04 - 1.51) log-rank $P=0.02)$. However, TREM2 expression has less influence on

156 Gastric cancer(Figure 3). We can also notice that the low expression of TREM2 predicts a 157 better OS in lung cancer and liver cancer, while a poor OS in breast cancer. The last, we analyzed relationships between TREM2 expression and prognostic values by using the GEPIA DATASET, which analyzing the RNA sequencing expression data of 33 types of cancer from TCGA (Supplementary Figure 1). Low TREM2 expression levels were associated with a better

161 prognosis of OS and DFS in LGG, LIHC, OS in COAD, and DFS in PRAD. Meanwhile, LOW TREM2 expression was correlated with poorer prognosis of OS in $\mathrm{CHOL}$, KIRP, and SKCM. 163 These results confirmed the prognostic value of TREM2 in some specific types of cancers, and that increased and decreased TREM2 expression have different prognostic value depending on the type of cancer.

The correlation between trem2 expression lever and clinicopathological features

As shown in the table 1, In KIRP, those who process following characteristics has a better prognosis:type2(HR=4.05, $\mathrm{P}=0.018), \mathrm{T} 2(\mathrm{HR}=6.95, \mathrm{P}=0.05), \mathrm{T} 2+\mathrm{T} 3(\mathrm{HR}=3.94, \mathrm{P}=0.02)$, stage $\| I(H R=4.44, P=0.01)$,stage $\|+\|(H R=4.95, P=0.0048)$, stage $\| I+I V(H R=2.61, P=0.0248)$, In age

172 and race, No significant correlation was found . moreover, the Under-expression of TREM2 173 benefited Female liver cancer patients of $\mathrm{OS}(\mathrm{HR}=4.44, \mathrm{P}=0.01)$, while benefited male of $174 \mathrm{PFS}(\mathrm{HR}=4.44, \mathrm{P}=0.01)$. High TREM2 $\mathrm{mRNA}$ expression was correlated with worse OS in stages 1752 to 4 , grades 2 to 3 , but was not associated with OS stage 1. 
179 The above results support the prognostic role of TREM2 in pan-cancer. Therefore, it is of 180 great significance to explore the relationship between TREM2 expression and immune 181 infiltration. TIMER got the TREM2 expression and immune infiltration level coefficient to discuss their correlation in 39 cancer types. The results indicated that TREM2 expression has significant correlations with tumor purity in 33 types of cancer. TREM2 expression has significant correlations with B cells, CD8+ T cells, CD4+ T cells, macrophages, neutrophils, and dendritic cells. The number of tumors is $21,23,33,34,28,34$, respectively. Then, we should decide on the tumor. On the one hand, the effect of tumor purity should be considered. On the other hand, it needs associating with TREM2 both in prognosis and immune infiltration. Based on the above, we chose to focus on LIHC and KIRP. Both for LIHC and KIRP, there is a strong correlation between TREM2 expression with the monocyte (LIHC: HR=0.554, $\mathrm{P}=7.64 \mathrm{e}$ -29 KIRP: HR=0.555, $P=1.53 e-21)$, and Dendritic cell(LIHC: $H R=0.53, P=5.48 e-26 \quad$ KIRP: $H R=0.645, P=1.52 e-31$ ), furthermore, the expression of TREM2 has significant weakly and modestly positive correlations with infiltrating levels of the B cell, CD8+ T cells, CD4+ T cells, and neutrophils in LIHC and KIRP, those results showed in figure4. Those results implicated that TREM2 plays a significant role in immune infiltration in LIHC and KIRP, especially macrophages and DCs.

TREM2 expression is associated with Immune Marker Sets

To study the relationship between TREM2 and the various immune infiltrating cells, we concentrated on the correlations between TREM2 and immune marker sets of assorted 
immune cells in KIRP and LIHC within the TIMER and GEPIA databases. Various immune marker genes of immune cells mentioned below were studied, such as CD8+ T cells, T cells

201 (general), B cells, monocytes, TAMs, M1 and M2 macrophages, neutrophils, and NK. Cells as well as DCs. Different functional T cells were also studied, included Th1 cells, Th2 cells, Tfh cells, Th17 cells, Tregs, and exhausted T cells. The results adjustment by purity revealed that the expression of TREM2 was significantly positively correlated with most immune marker sets in KIRP and LIHC, Specifically monocytes andDCs. It is worth noting that some difference in results between KIRP and LIHC (Table 2 and Figures 5). We found that TREM2 expression is associated with CD8 + T cell markers, challenging to be detected in KIRP.

Similarly, the expression of TREM2 has strong correlations with Treg and T cell exhaustion in LIHC. However, these two types were less significant in another. The details are as follows. For CD8+T cells, we can find a positive correlation between TREM2 expression and CD8A and CD8B in KIRP. For DC cell, including HLA-DPB1, HLA-DQB1, HLA-DRA, HLA-DPA1, CD11c, those markers are positively strong related with TREM2 expression both in KIRP and LIHC. for Treg cells and T cell exhaustion, In KIRP, TREM2 has an only positive correlation with the TIMTGFB1, PD-1, CTLA4, TIM-3. DCs play a central role in regulating the balance between CD8

216 T cell immunity and tumor antigen tolerance. It can promote activated CD8+ T cells and be 217 controlled by TME, enabling tumor development[19]. Treg cells participate in the occurrence 218 and development of tumors by inhibiting anti-tumor immunity[20]. 'T cells exhaustion ' refers to the gradual deterioration of CD8 $+\mathrm{T}$ cells function when cells are exposed to antigens or inflammatory signals for a long time. The expression of multiple inhibitory receptors (such as 
PD-1 and LAG3) is the characteristic of exhausted T cells[21]. We further verify the results with the GEPIA database. We found that the results are similar to those in TIMER (Table 3). These results also reveal that TREM2 plays a vital role in the microenvironment.

\section{Discussion}

For a long time, people have devoted themselves to studying the role of immune receptor TREM2 in human neurodegenerative diseases. In recent years, many researchers have tried to explore the relationship between TREM2 and tumors. TREM2 has been confirmed as a marker for tumor-associated macrophages (TAMs) and monocytes [22]. Research reports that TREM2 is a Tumor Suppressor in Colorectal Carcinoma and Exert negative immunomodulatory function in lung cancer[23] [24].however, the role of TREM2 expression has not been elucidated in KIRP.

In this study, we explore what role TREM2 played in tumor prognosis and tumor microenvironment. We use Oncomine datasets to analyze TREM2 expression via three datasets to find further information about prognosis. Our results indicated that TREM2 mRNA expression is higher in bladder cancer, brain and CNS cancer, breast cancer, cervical cancer, colorectal cancer, esophageal cancer, stomach cancer, head and neck, kidney cancer, pancreatic cancer, and prostate cancer. But it is lower in lung cancer. Analysis of the TCGA data showed that TREM2 expression was higher in BLCA、BRCA、CESC、CHOL、COAD、 GBM、HNSC、KICH、KIRC、KIRP、LIHC、PRAD、STAD、THCA、UCEC, but the lower expression in LUAD and LUSC compared with normal adjacent tissues. We can easily find that the results are highly consistent. By analyzing the results from several datasets, we found that the high 
expression of TREM2 may indicate poor prognosis in colorectal cancer, ovarian cancer, lower-

244 grade Glioma, and liver cancer. We also found that TREM2 overexpression may predict a

245 better prognosis in KIRP. We also pay more attention to Kidney renal papillary cell carcinoma

246 (KIRP) patients, meanwhile using liver hepatocellular carcinoma (LIHC)as the control. The

247 relationship between TREM2 mRNA level and clinicopathological features in patients with Kirp

248 and LIHC was investigated using bioinformatics. The result suggested that low TREM2

249 expression levels were significantly correlated with tumor type, higher T stage, and higher

250 TNM stage in KIRP. On the contrary, high TREM2 expression levels were significantly

251 associated with tumor type, higher T stage, higher TNM stage, and higher GRADE in LIHC. It

252 has been described in articles that TREM2 is a new type of tumor suppressor gene for liver

253 cancer, and the decreased expression of TREM2 in HCC patients is associated with poor

254 prognosis[25] the results are consistent. We conclude that high TREM2 expression may can

255 as an independent prognostic factor for KIRP and liver patients.

256 Finally, we use TIMER to explore the relationship between TREM2 and diverse immune

257 infiltration levels in cancer. Our results indicate a strong positive correlation between the

258 expression level of TREM2 and the level of macrophages and dendritic cell infiltration, a

259 modest positive correlation in other infiltration levels (B cells, CD8+ T cells, CD4+ T cells, and

260 neutrophils) both in KIRP and LIHC. To further explore the reasons for the different results,

261 We investigated the correlation between immune cell marker genes and TREM2 expression.

262 The same point is that the rise in expression of TREM2 is positively associated with the 263 expression of markers of T cell, monocyte, TAM, m2 Macrophage, and DC cell both in KIRP 264 and LIHC. The slightly noticeable difference is that a modest positive correlation between the 
266 T cell exhaustion markers expression in KIRP.

267 Furthermore, The rise in expression of TREM2 is positively associated with the expression of markers of Treg and T cell exhaustion in LIHC. These results from TIMER datasets are after purity correction. We further verified the accuracy of the results with the GEPIA database. Some researches indicated that high TREM2 expression predicts poor prognosis

271 in other tumors like Glioma, mammary tumors, and colorectal cancer. According to our result,

272 We still have reason to believe that high TREM2 expression has a better prognosis in KIRP.

273 These results may be related to the differential immune infiltration of different tumors. Also,

274 Different tumors have other mechanisms that play a significant role. It was already mentioned 275 above that the effects of TREM2 in modulating tumor immunology may be achieved by DC 276 cell and M2 macrophage in KIRP and LIHC. Dendritic cells (DC) play a central role in regulating 277 the balance between CD8 $\mathrm{T}$ cell immunity and tumor antigen tolerance, while M2 278 macrophages suppress intratumoral CD8+ T cell recruitment [19]. According to the difference 279 of results, we can boldly speculate that CD8 T cells activated by DC through cross280 presentation of exogenous antigen may be dominant in KIRP. At the same time, M2 281 macrophage and TREG may play the leading role. It may be the starting point for our next 282 experimental study.

283 In summary, increased TREM2 expression correlates with a better prognosis in KIRP. Both in $284 \mathrm{KIRP}$ and LIHC, TREM2 expression potentially contributes to the regulation of T cell, 285 monocyte, TAM, M2 macrophage, and DCs. Besides, in KIRP, TREM2 expression may 286 participate in the regulation of CD8+Tcell in KIRP while regulating Treg in LIHC. Therefore, 
prognosis biomarker in KIRP.

\section{References:}

[1].Akhtar M, Al-Bozom IA, Al Hussain T. Papillary Renal Cell Carcinoma (PRCC): An

Update.J. Adv Anat Pathol. 26(2):124-132(2019).

[2]. Sheng IY, Rini BI. Immunotherapy for renal cell carcinoma. J. Expert Opin Biol Ther. 19(9):897-905(2019).

[3]. Dutcher JP, Flippot R, Fallah J.et al. On the shoulders of giants: the evolution of renal

cell carcinoma treatment-cytokines, targeted therapy, and immunotherapy.J. Am Soc Clin

Oncol Educ Book. 40:1-18(2020).

[4]. Deleuze, A., et al., Immunotherapy in Renal Cell Carcinoma: The Future Is Now. Int J Mol Sci,

[5]. Santoni, M., et al., Immunotherapy in renal cell carcinoma: latest evidence and clinical implications.

Drugs Context, 2018. 7: p. 212528.

[6]. Carmona, S., et al., The role of TREM2 in Alzheimer's disease and other neurodegenerative

disorders. Lancet Neurol, 2018. 17(8): p. 721-730.

304

[7]. Ulland, T.K. and M. Colonna, TREM2 - a key player in microglial biology and Alzheimer disease.

Nat Rev Neurol, 2018. 14(11): p. 667-675.

[8]. Deczkowska, A., A. Weiner and I. Amit, The Physiology, Pathology, and Potential Therapeutic

Applications of the TREM2 Signaling Pathway. Cell, 2020. 181(6): p. 1207-1217. 
Pathol, 2018. 72: p. 91-99.

310 [10]. Wang, X.Q., et al., Overexpression of TREM2 enhances glioma cell proliferation and invasion: a 311 therapeutic target in human glioma. Oncotarget, 2016. 7(3): p. 2354-66.

312 [11]. Tang, W., et al., TREM2 acts as a tumor suppressor in hepatocellular carcinoma by targeting the PI3K/Akt/beta-catenin pathway. Oncogenesis, 2019. 8(2): p. 9.

[12]. Katzenelenbogen, Y., et al., Coupled scRNA-Seq and Intracellular Protein Activity Reveal an Immunosuppressive Role of TREM2 in Cancer. Cell, 2020. 182(4): p. 872-885.e19.

[13]. Rhodes, D.R., et al., Oncomine 3.0: genes, pathways, and networks in a collection of 18,000 cancer gene expression profiles. Neoplasia, 2007. 9(2): p. 166-80.

[14]. Mizuno, H., et al., PrognoScan: a new database for meta-analysis of the prognostic value of genes. BMC Med Genomics, 2009. 2: p. 18.

[15]. Tang, Z., et al., GEPIA: a web server for cancer and normal gene expression profiling and interactive analyses. Nucleic Acids Res, 2017. 45(W1): p. W98-W102.

[16]. Menyhart, O., A. Nagy and B. Gyorffy, Determining consistent prognostic biomarkers of overall survival and vascular invasion in hepatocellular carcinoma. R Soc Open Sci, 2018. 5(12): p. 181006. data via the Xena platform. J.Nat Biotechnol. 38(6):675-678(2020).

[18]. Li T, Fan J, Wang B, et al. TIMER: A Web Server for Comprehensive Analysis of Tumor327 Infiltrating Immune Cells. J.Cancer Res. 77(21):e108-e110(2017).

[19]. Fu C, Jiang A. Dendritic Cells and CD8 T Cell Immunity in Tumor Microenvironment. J.Front Immunol. 20;9:3059(2018). 
331 therapeutic target?J. Cancer Sci. 110(7):2080-2089(2019).

332 [21]. Kurachi M. CD8+ T cell exhaustion. J.Semin Immunopathol. 41(3):327-337(2019).

333 [22]. Nakamura K, Smyth MJ. TREM2 marks tumor-associated macrophages. J.Signal 334 Transduct Target Ther. 5(1):233(2020).

335 [23]. Yao Y, Li H, Chen J, et al. TREM-2 serves as a negative immune regulator through Syk 336 pathway in an IL-10 dependent manner in lung cancer. J.Oncotarget. 7(20):29620-34(2016). 337 [24]. Kim SM, Kim EM, Ji KY, et al. TREM2 Acts as a Tumor Suppressor in Colorectal 338 Carcinoma through Wnt1/ $\beta$-catenin and Erk Signaling.J. Cancers (Basel). 11(9):1315(2019).

339 [25]. Esparza-Baquer A, Labiano I, Sharif O, et al. TREM-2 defends the liver against 340 hepatocellular carcinoma through multifactorial protective mechanisms. J.Gut.:gutjnl-2019$341319227(2020)$.

342 
Figures

A

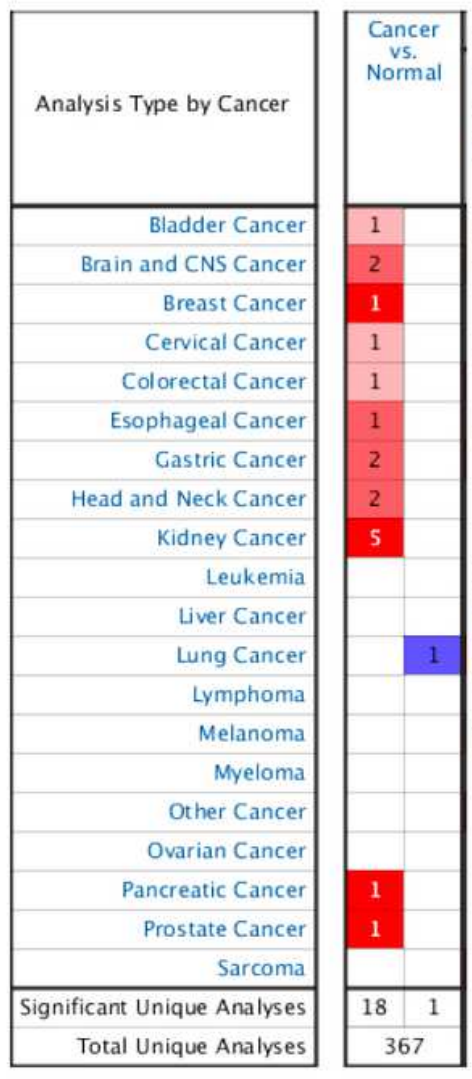

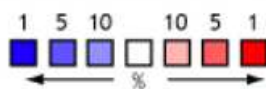

Gene rank percentile(\%)
B

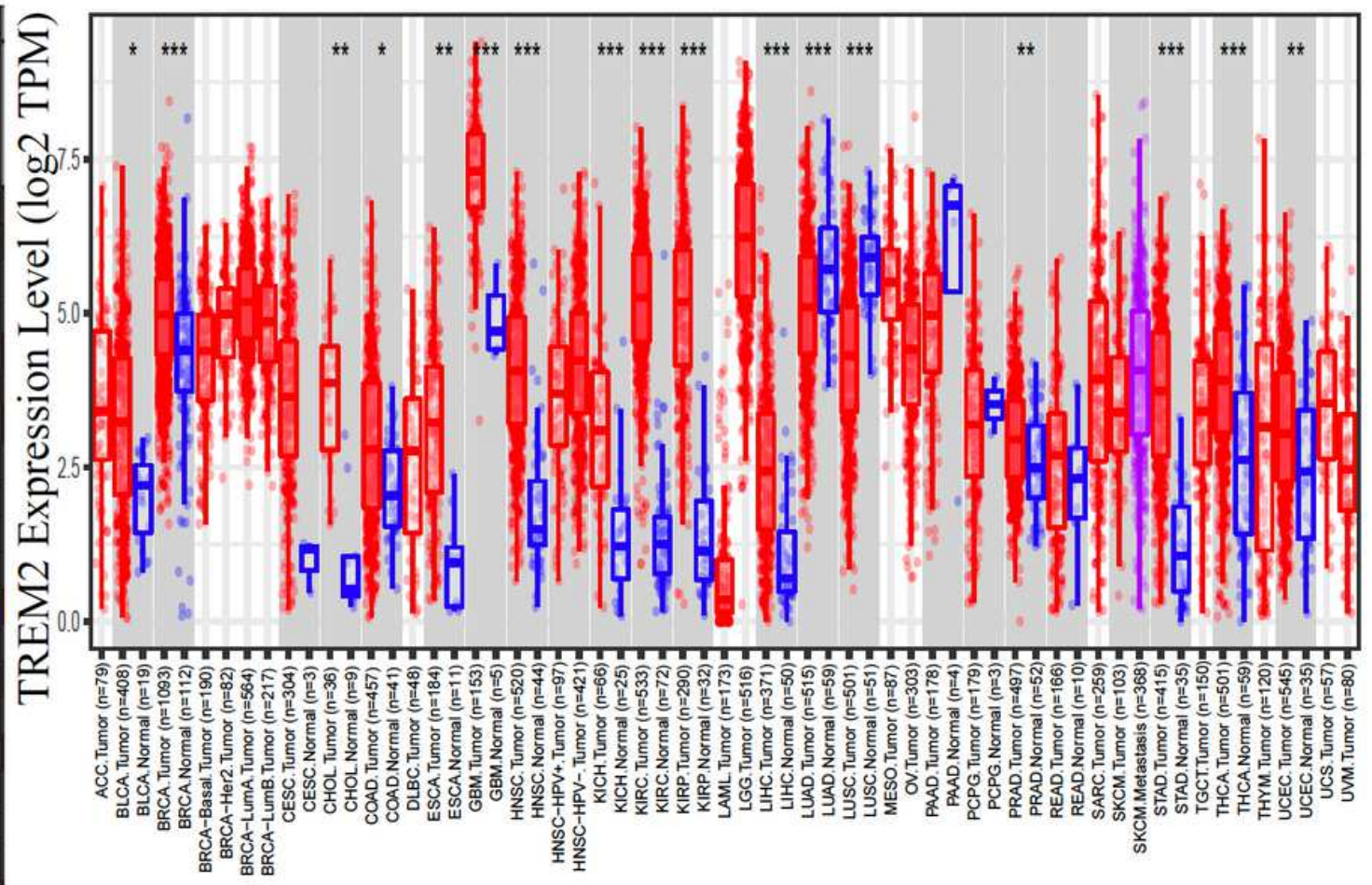

Figure 1

Caption not available with this version 
A

Lung cancer,GSE31210,RFS, $\mathrm{HR}=1.88, \operatorname{coxp}=<0.001$.

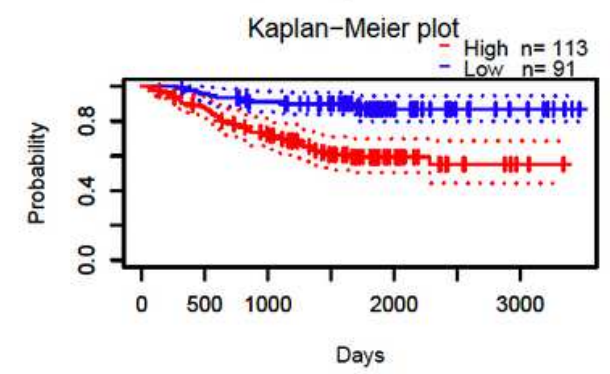

D

Lung cancer,GSE11117,OS, $\mathrm{HR}=0.55, \mathrm{coxp}=0.008$.

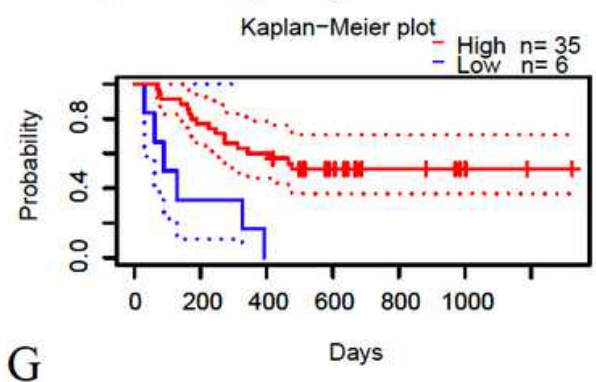

Breast cancer,GSE19615, DMFS, HR $=0.32$, Coxp $=0.029$

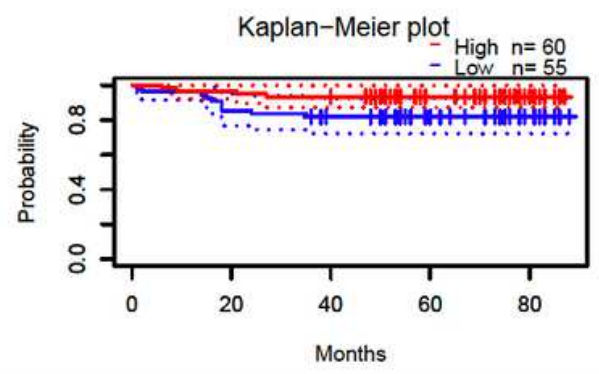

B

Lung cancer, GSE1321, OS, $\mathrm{HR}=0.37$, Cox $\mathrm{p}=<0.001$.

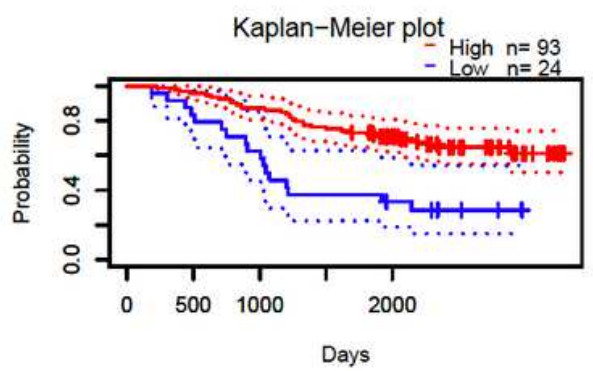

E

Lung cancer,GSE13213,OS, $\mathrm{HR}=0.72, \mathrm{Coxp}=0.019$

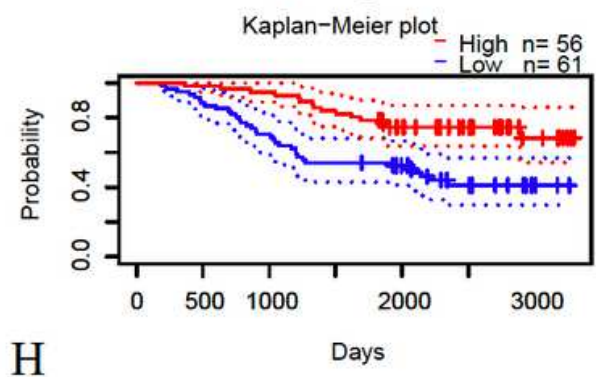

Breast cancer,GSE1379, RFS, HR=2.15, $\operatorname{coxp}=0.044$

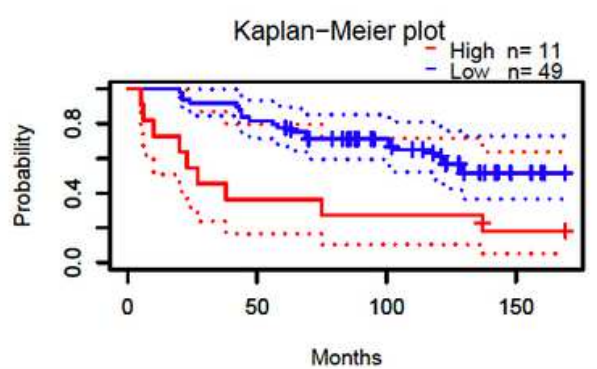

C Lung cancer,GSE31210,OS. $\mathrm{HR}=2.21, \mathrm{Coxp}=0.0014$.

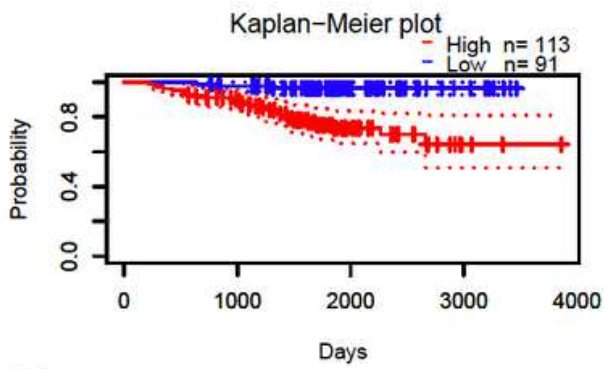

$\mathrm{F}$

Colorectal cancer,GSE14333

DFS, $H R=1.23, \operatorname{coxp}=0.026$

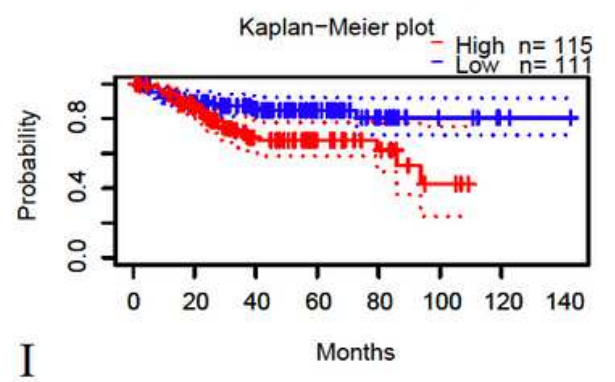

Colorectal cancer,GSE17537, DFS, $\mathrm{HR}=2.07, \mathrm{Coxp}=0.046$

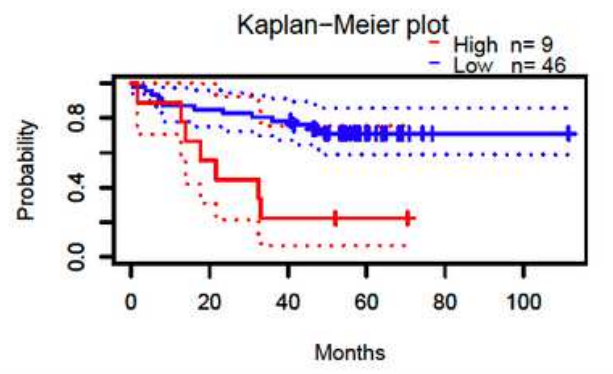

Figure 2

Caption not available with this version 


\section{A Breast cancer,OS B Breast cancer,PFS}
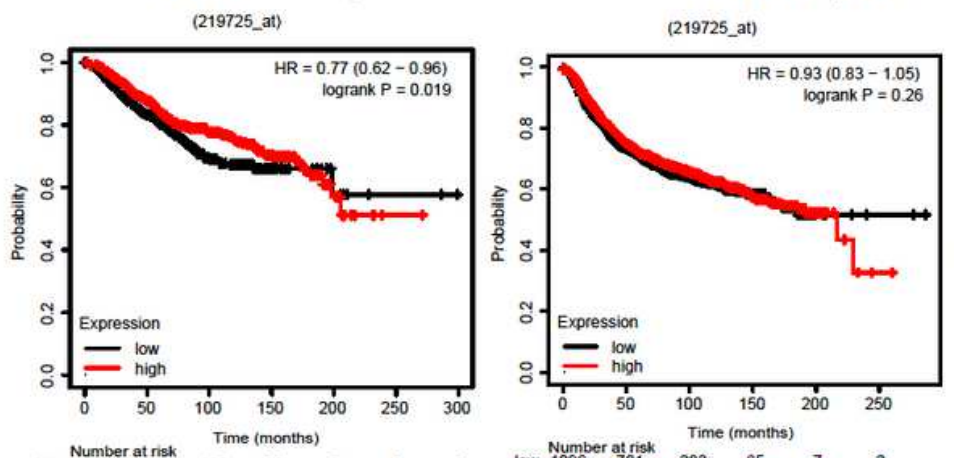

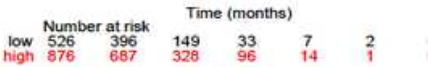

\section{E \\ Lung cancer, OS}

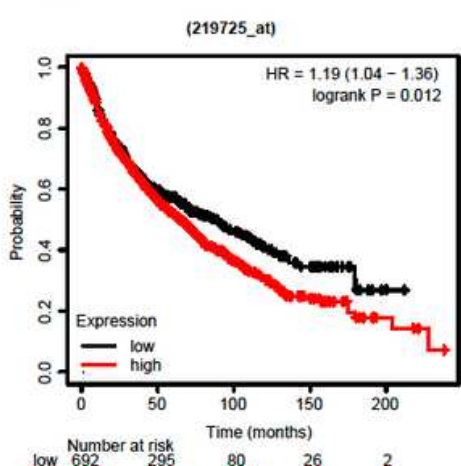

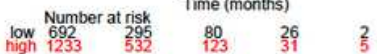

C Gastric cancer,OS

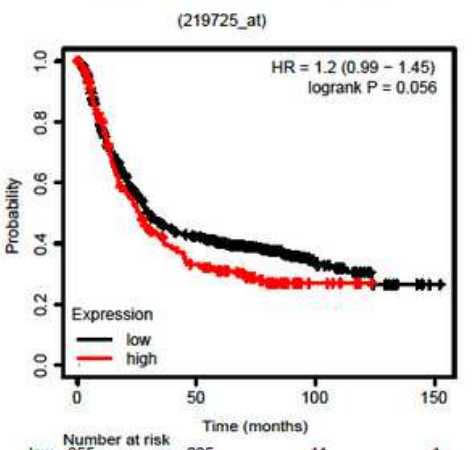

\begin{tabular}{lll} 
low Number at risk & \multicolumn{2}{c}{ Time (months) } \\
high 225 & 235 & 41
\end{tabular}
D Gastric cancer,PFS

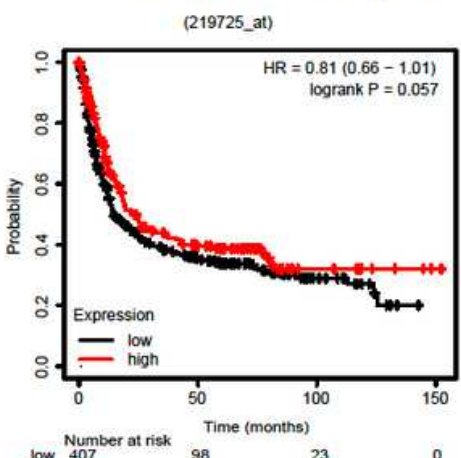

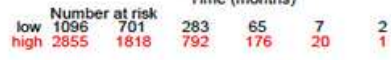
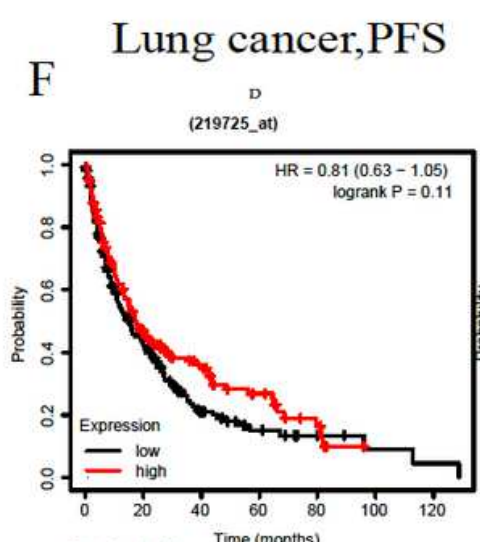

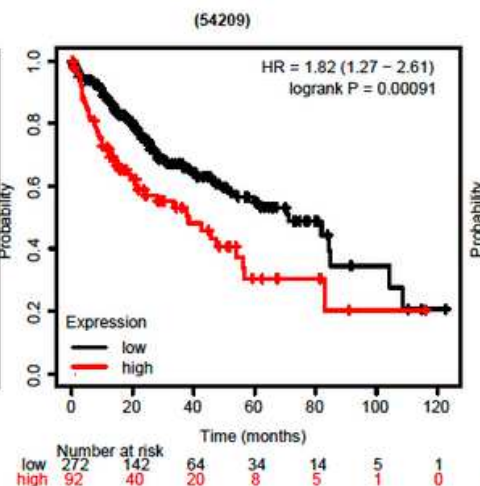

\section{Giver cancer,OS}

\section{$\mathrm{H}$ \\ Liver cancer,PFS}

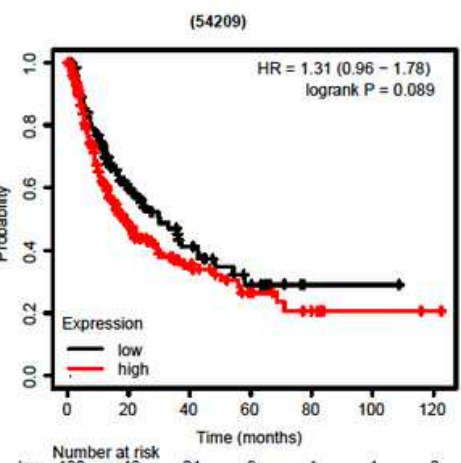

I

Ovarian, OS

$\mathrm{J}$

\section{Ovarian,PFS}
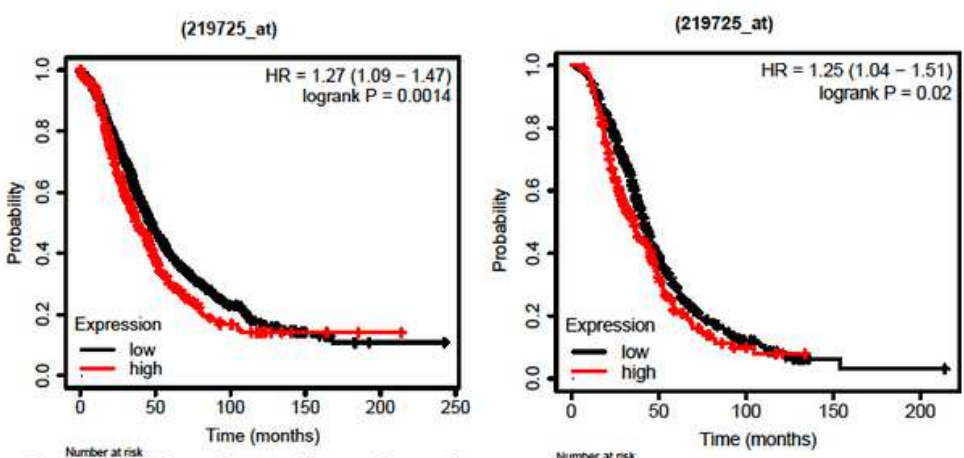

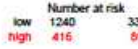

\section{Figure 3}

Caption not available with this version 


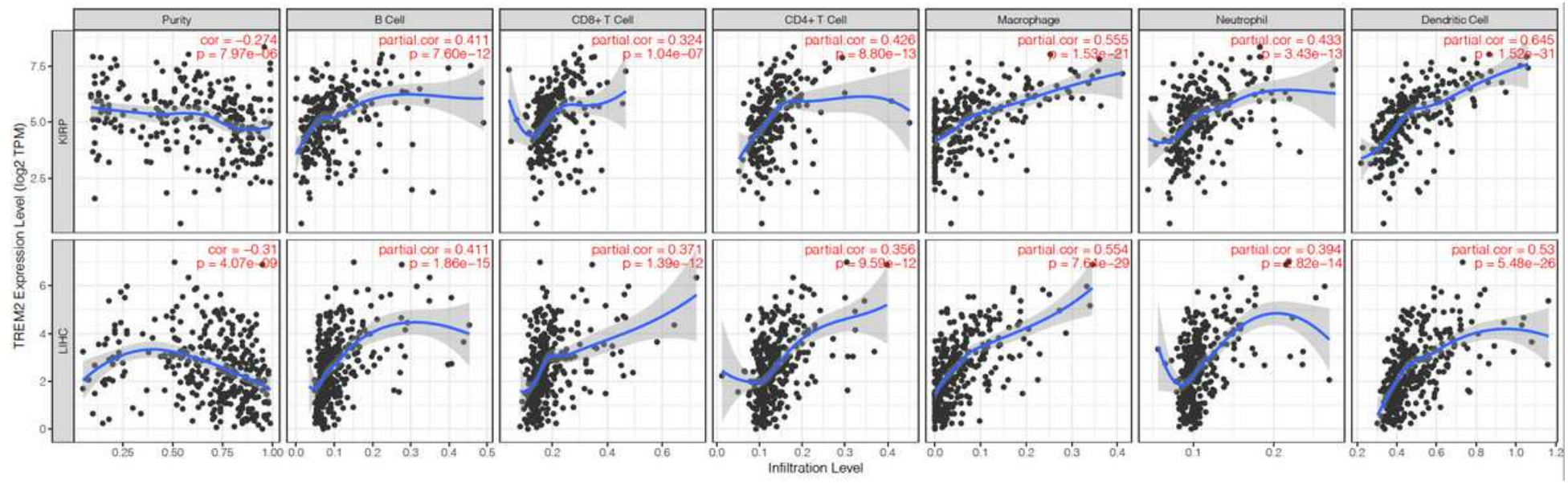

Figure 4

Caption not available with this version 

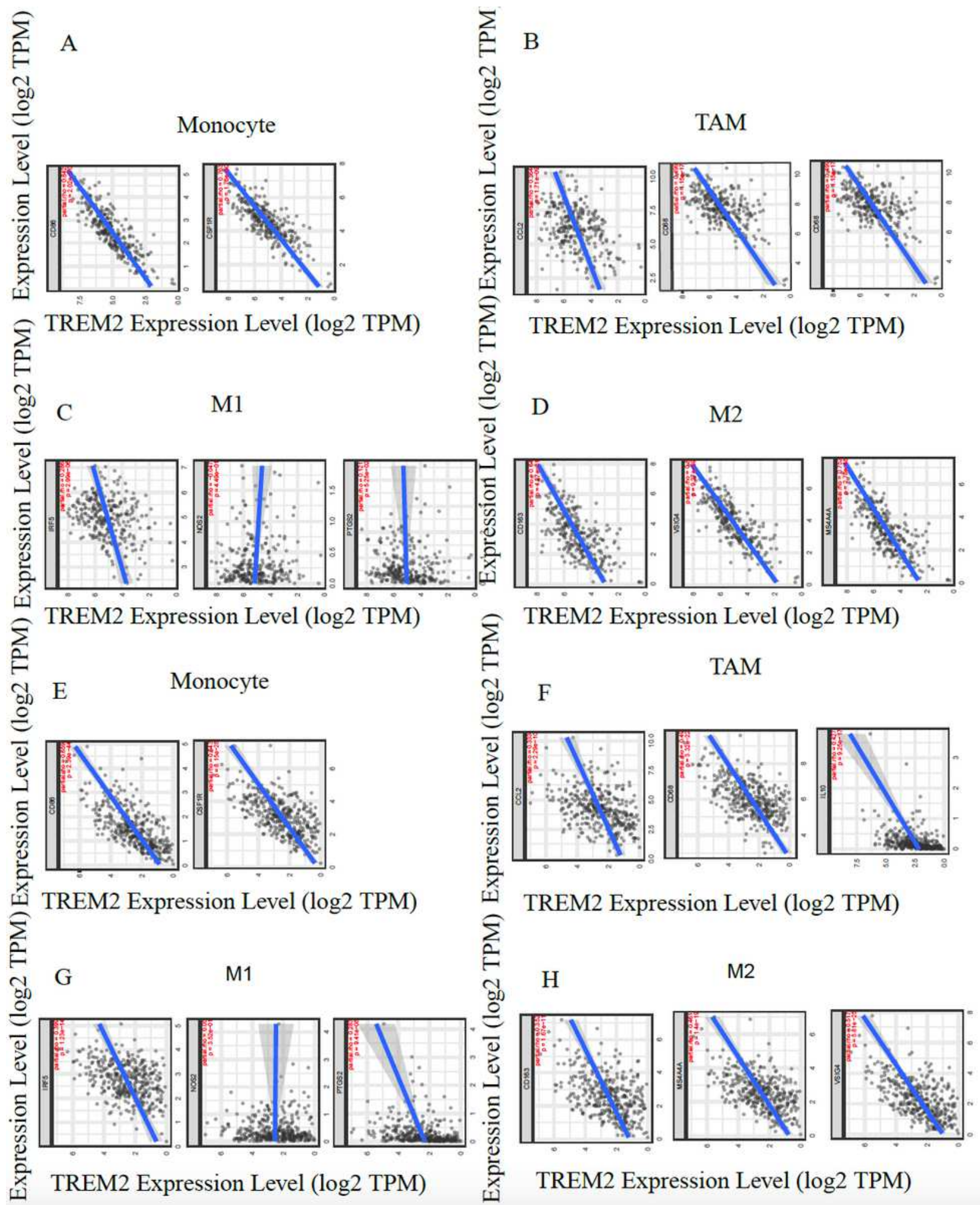

\section{Figure 5}

Caption not available with this version

\section{Supplementary Files}

This is a list of supplementary files associated with this preprint. Click to download. 
- Table1.xlsx

- Table2.xIsx

- Table3.xlsx

- SupplementaryFigure1.1.pdf

- SupplementaryFigure1.2.pdf

- SupplementaryFigure1.3.pdf

- SupplementaryFigure1.4.pdf

- SupplementaryFigure2.pdf

- SupplementaryTable1.xIsx 